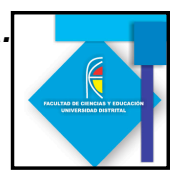

\title{
A PROPÓSITO DEL PRINCIPIO DE CONSERVACIÓN DE LA ENERGÍA: UNA PROPUESTA DE FORMALIZACIÓN EN LA ENSEÑANZA DE LA FÍSICA DESDE UN ANÁLISIS HISTÓRICO Y EPISTEMOLÓGICO DE LA PERSPECTIVA DE MAYER \\ ABOUT THE PRINCIPLE OF ENERGY CONSERVATION: A PROPOSAL TO FORMALIZE EDUCATION IN PHYSICS FROM ANHISTORICAL AND EPISTEMOLOGICAL MAYER'S PERSPECTIVE
}

\author{
Alba Denis Arboleda Correa ${ }^{1}$ \\ Mónica Xiomara Díaz Valencia ${ }^{2}$ \\ Yirsen Aguilar Mosquera ${ }^{3}$
}

\section{Resumen}

En el análisis realizado en ciertas investigaciones se logra evidenciar que algunas dificultades de los estudiantes en física, están estrechamente relacionadas con la relación que establecen entre el conocimiento común y el conocimiento científico. Usualmente los estudiantes explican los fenómenos desde el conocimiento común, es decir desde lo que se ha aprendido en la cotidianidad, como caso particular se puede referenciar situaciones físicas que se resuelven con la aplicación del Principio de Conservación de la Energía (Pozo, 2000). En estas situaciones resulta problemática la relación que establecen entre los conceptos de trabajo, masa, transferibilidad, calor y fuerza, entre otros. En la relación de trabajo y energía, el trabajo se conceptualiza en términos de la energía y la energía en términos del trabajo, es decir, se plantea una circularidad entre estos dos conceptos. Igualmente, los estudiantes asignan un carácter material a la energía, se confunden las formas de energía con sus fuentes, se le asigna sustancialidad al calor y no se tienen en cuenta procesos de transformación, transferencia y degradación de la energía.

\footnotetext{
${ }^{1}$ Estudios Culturales sobre las Ciencias y su Enseñanza - ECCE-Facultad de Educación, Universidad de Antioquia. Medellín, Colombia. aaaarbol@gmail.com

${ }^{2}$ Estudios Culturales sobre las Ciencias y su Enseñanza - ECCE-Facultad de Educación, Universidad de Antioquia. Medellín, Colombia. mxdiaz84@gmail.com

${ }^{3}$ Estudios Culturales sobre las Ciencias y su Enseñanza - ECCE-Facultad de Educación, Universidad de Antioquia. Medellín, Colombia, yirsena@yahoo.es
} 


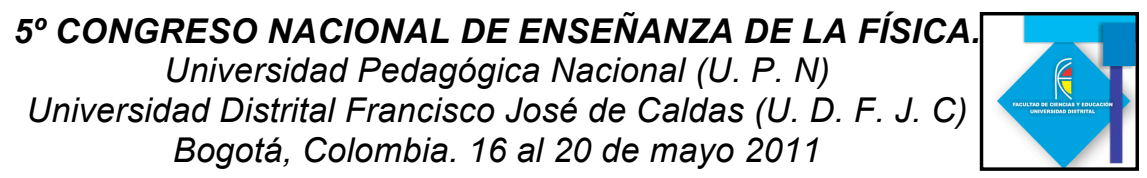

Con la intención de construir rutas conceptuales alternativas para la enseñanza de la mecánica, se realiza un análisis histórico epistemológico de la manera como Mayer configura y presenta el Principio de Conservación de la Energía, expuesto en su obra titulada Observaciones sobre las energías de la naturaleza inorgánica (1842). En este análisis se muestra cómo Mayer establece el Principio de Causalidad como una función (las causas = efectos=causas), considera las causas como entes cuantitativamente indestructibles y cualitativamente convertibles y partir de de estas consideraciones plantea que las energías son entes indestructibles y convertibles. Teniendo en cuenta estas consideraciones se asume la indestructibilidad y convertibilidad como Principios estructurante en la Conservación de la Energía y se re significan los conceptos de trabajo, fuerza y transferibilidad.

Finalmente, a partir del análisis de la propuesta de formalización de Mayer y los modelos explicativos de cinco casos del grado once de la Institución Educativa Comercial de Envigado, se plantean unas implicaciones didácticas donde se consideran actividades que posibilitan precisar sobre los conceptos articuladores del Principio de Conservación de la Energía.

Palabras clave: Conservación, Energía, Convertibilidad, Mayer, Indestructibilidad, Causalidad.

\section{Abstract}

In the analysis done in some researches, shows that some students' difficulties in physics, are closely related to the relationship established between common knowledge and scientific knowledge. Usually, students explain the phenomena from common knowledge, that is, from what they have learnt in their daily life, as a particular case, we can make reference to physical situations that are solved by the application of the Principle of Energy Conservation (Pozo, 2000). In these situations, it is kind of problematic the relationship established between the concepts of work, mass, portability, warmth and strength, among others. In relation to work and energy, the work is conceptualized in terms of energy and energy in terms of work; that means that there is a circularity arised between these two concepts. Also, students assigned a material character to energy, mixed forms of energy with its sources, it is given substantiality to heat and does not take into account processes of transformation, transfer and degradation of energy.

With the intention to build some conceptual alternative routes for teaching of the mechanics, it is done a historical epistemological analysis on Mayer's way, how he sets up and presents the Principle of Energy Conservation, stated in his work named Observations on the energies of inorganic nature (1842). This analysis shows how Mayer establishes the principle of causality as a function (cause $=$ effect $=$ cause), he considers the causes how entities indestructible quantitatively and convertible qualitatively and from these considerations, he suggests that energies are indestructible and convertible entities. Taking into account these considerations, it is assumed the indestructibility and convertibility as structured Principles in Energy Conservation and they remean the terms of work, power and portability. 


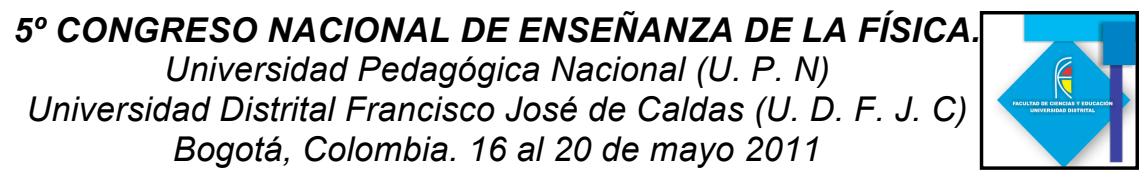

Finally, from the analysis of the Mayer formalization proposal and explanatory models of five cases of eleventh grade of Institución Educativa Comercial of Envigado, it is presented some pedagogical implications which are considered activities that enable on the principle Energy Conservation concepts.

Keywords: Conservation, Energy, Convertibility, Mayer, indestructibility, Causality.

\section{Una mirada del principio de conservación de la energía (PCE) en el contexto de la enseñanza}

En el análisis de investigaciones se ha logrado evidenciar algunas dificultades en la constante búsqueda del hombre por dar respuesta a sus inquietudes, en donde se establecen relaciones entre el conocimiento común y el conocimiento científico. En este sentido, es importante resaltar que los modelos explicativos de los estudiantes son creados a partir de analogías que le permiten explicaciones válidas en su contexto, sin embargo debe reconocerse que en muchas ocasiones esos modelos explicativos riñen con los planteados en la ciencia y a veces obstaculizan el aprendizaje de la misma. Es por esto que a veces la observación y análisis de los procesos primarios y el transcurso de los eventos, solo se centra en los estados finales del fenómeno, dejando de lado aspectos estructurantes en la ocurrencia de estos.

Otra dificultad que presentan los estudiantes es que no reconocen la conservación de la energía como un principio universal, no consideran que en distintas condiciones sucedan determinados fenómenos, por medio de los cuales comprenderían mejor hechos particulares, de aquí que no se adelanten a los sucesos, y así lograr predecirlos. Por lo general los alumnos solo aceptan la existencia de aquello que pueden observar directamente, por lo tanto las dificultades en el aprendizaje de la física se deben a que esta parece ser abstracta y difícil de imaginar, sumado a esto, la objetividad por la que se propende en el aula de clases parece no estar presente, pues sin argumentos, no se da anclaje del discurso y mucho menos del lenguaje científico, a esto se añade en ocasiones, falta de interés, desconocimiento de bases conceptuales, entre otros ( Henao y Stipcich, 2008)

En este sentido, es pertinente hablar de investigaciones como las de Solbes y Tarín (1998) en las que indican que los estudiantes solo observan los cambios en cuanto fenómenos mecánicos (movimiento), dejando de lado la importancia de otros fenómenos como los termodinámicos. En estas situaciones resulta problemática la relación que establecen entre los conceptos de trabajo, masa, transferibilidad, calor y fuerza, entre otros. En la relación de trabajo y energía, el trabajo se conceptualiza en términos de la energía y la energía en términos del trabajo, es decir, se plantea una circularidad entre estos dos conceptos (Carmona, Amaya. 2007). Igualmente, los estudiantes asignan un carácter material a la energía, se confunden las formas de energía con sus fuentes (Pérez y Galeano 2008), se le asigna sustancialidad al calor (Albert, 1978; Erickson, 1979, 1980) y no se tienen en cuenta procesos de transformación, transferencia y degradación de la energía. 


\section{Dificultades del PCE en aula.}

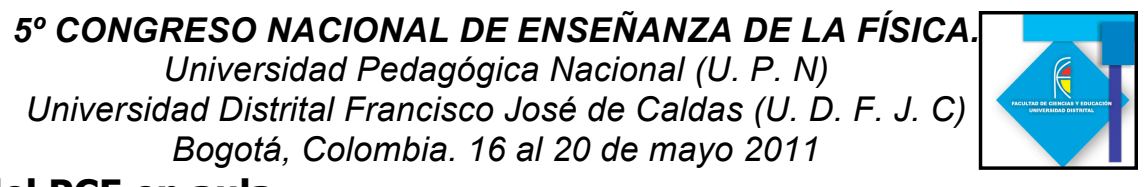

En el contexto del aula de clases se ha podido identificar la no interrelación entre causa y efecto, los estudiantes parecen olvidar que toda causa conlleva a un efecto y este a su vez otro efecto o efectos, además de asumir como perdida aquello que no pueden percibir, dejando entonces como resultado, un sin sentido en sus experiencias, pues aquello que no ven, simplemente se destruye y no se conserva.

Conociendo lo anterior, se hace necesario introducir en las aulas de clase la teoría de sistemas, esta teoría, permite conocer el conjunto de las interacciones que al estar sus partes ligadas unas con otras, dan cuenta de su estado y de las modificaciones que este sufre, las condiciones de un cuerpo no cambian solas, el cuerpo no hace nada por sí mismo, un efecto no nace de la nada, postura tomada desde Robert Mayer, quien desde su principio causa aequat effectum, muestra que es necesario pensar que un efecto fue creado por una causa; de esta forma se hace evidente la idea de observar cada elemento de los diferentes sistemas, y así lograr una relación entre fenómenos, que a su vez, sea coherente y forme parte de la correspondiente estructura (Aguilar, Restrepo, Mejía, 2002), cuando se busca la conexión del fenómeno y este a su vez se ve reflejado en otro.

Se hace pertinente acceder a la historia y la epistemología, rescatando la manera en la cual se pensó y planteo desde sus inicios el PCE, analizando pensadores y científicos como Robert Mayer, con su obra Observaciones sobre las energías de la naturaleza inorgánica (1842), de la cual se logro retomar diferentes análisis en los cuales intento igualar la noción de fuerza con materia, propendiendo por dar explicación al Principio de Causalidad como una función (las causas = efectos=causas), quien considero las causas como entes cuantitativamente indestructibles y cualitativamente convertibles. Teniendo en cuenta el principio de causalidad se hace posible asumir la indestructibilidad y convertibilidad como ejes estructurantes en el PCE y así resignificalo, para facilitar la comprensión de procesos tales como transformación, transferencia y degradación de la energía.

\section{Importancia de la Historia y la Epistemología en la enseñanza del principio de conservación de la energía}

Es evidente la importancia no sólo de conocer la historia de la ciencia, sino su relación con la epistemología, la cual permite contextualizar la historia que subyace a las teorías de una disciplina, y permite promover no sólo la comprensión de lo actual, sino que también proporciona nuevas posibilidades en los modos de significar el conocimiento y los fenómenos físicos, estos últimos con fines didácticos. (Mach 1883).

Las posibilidades que ofrecen la historia y la epistemología de las ciencias en el PCE promueven acercamientos a una ciencia más crítica, más reflexivas y más humanizada. Al acceder a la historia y la epistemología, se logra rescatar la manera en la cual se pensó y planteo desde sus inicios este principio, analizando pensadores y científicos como Robert Mayer, con su obra Observaciones sobre las energías de la naturaleza inorgánica (1842), de la cual se logro retomar diferentes análisis en los cuales intento igualar la noción de fuerza con materia, propendiendo por dar explicación al Principio de Causalidad como una función (las causas $=$ efectos=causas), quien considero las causas como entes 


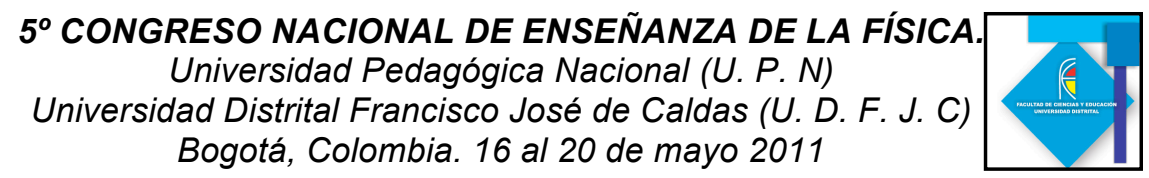

cuantitativamente indestructibles y cualitativamente convertibles.

Teniendo en cuenta el principio de causalidad se hace posible asumir la indestructibilidad y convertibilidad como

ejes estructurantes en el PCE y así resignificalo, para facilitar la comprensión de procesos tales como transformación, transferencia y degradación de la energía.

\section{Transformación, transferencia y degradación de la energía, resignificándolas desde el Principio de convertibilidad e indestructibilidad}

En las experimentaciones que realizó Robert Mayer en torno a la convertibilidad de fenómenos propone como un cambio de una clase de energía, puede generar un cambio y este cambio genera otra clase de energía, todo lo anterior explicado através del principio de effectum causa aequat.

Entendiéndose la convertibilidad como: "Si la causa $\mathbf{c}$ ha producido un efecto e siendo este efecto igual a la causa, por lo tanto, c ha dejado de existir en su forma inicial y se ha convertido en e, que es el efecto de esa conversión.

Después de la producción de un efecto e, y permaneciera aún la causa c, en todo o en parte, queriendo decir con esto que si existiese todavía restos de la causa, esta se puede convertir en otro tipo de efecto y a la causa que permanece le correspondería convertirse en otro efecto $u$ otro efecto de menor tamaño, así $e<c$ no siendo el único que pueda generar esta causa, por tanto c debería ser mayor que e (c > e) y así cada causa que subsista desencadena un efecto adicional.

Como consecuencia, al permanecer la causa c en todo o en parte, debe haber otros efectos, correspondientes a la causa que aún queda, ya que no es la misma, indicando con esta formulación que la causa no desaparece en su totalidad si no que el efecto es el que aparece en variedad, así se plantea entonces que: sí e es entonces causa a su vez de otro efecto $\mathbf{f}$, se tiene que $\mathbf{e}=\mathbf{f}$, por tanto $\mathbf{c}=\mathbf{e}=\mathbf{f} . . .=\mathbf{c}$. En esta cadena de causas y efectos, dentro de la ecuación resultante de igualdad, un término o una parte de él, nunca pueden ser o hacerse igual a cero", en otras palabras es posible de ser transformado, tranferido y degradado

De lo anterior se toma este al principio para darle una correspondencia con lo existente actualmente donde se establece que: la "energía no puede ser creada ni destruida", ya que desde estas razones solo puede ser causa de otro efecto, que desencadena en una serie de fenómenos que en sumatoria la contienen en su totalidad o parcialmente.

\section{Referencias bibliográficas}

Aguilar Mosquera, Y., Restrepo Cadavid, T y Mejía, R. (2002). El movimiento desde la perspectiva de sistema, estado y transformaciones. Facultad de Educación Especialización en ciencias experimentales

Cassire, E. (1979). Fin y método de la física teórica. 


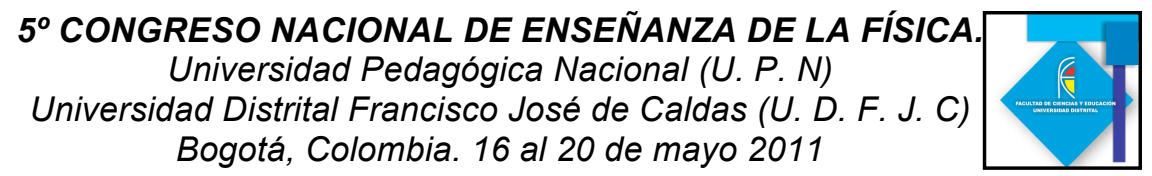

Ericzon, G.L.(1979,1980). Children's Conceptions of Heat and

Temperature. Science Education, 63, pp. 221-230.

Henao, B. L. y Stipcich, M. S. (2008). Educación en ciencias y argumentación: la perspectiva de Toulmin como posible respuesta a las demandas y desafíos contemporáneos para la enseñanza de las Ciencias Experimentales.

Mayer, R. J. Onthe forces of inorganic nature. Reprinted from R. B. Linsay, Men of Physics, Oxford, 1973, pp. 277- 283.

Moureen Pérez, J. y Galeano Marín, J. D. (2008). Recontextualización del principio de conservación de la energía desde una perspectiva histórico epistemológica: una propuesta para la enseñanza y el aprendizaje. Monografía. Facultad De Educación Universidad De Antioquia. Medellín.

Pozo, J. I. y Gómez Crespo, M. A. (1998). Aprender y enseñar ciencia: Del conocimiento cotidiano al conocimiento científico. Ed. Morata, Madrid. Pp. 205-262.

Solbes, J. y Tarín, F. (1998). Algunas dificultades en torno a la conservación de la energía. Enseñanza de las ciencias, 16(3), 387-397. 